\title{
Vertical integration of ultrafast semiconductor lasers
}

\section{Journal Article}

Author(s):

Maas, Deran; Bellancourt, A.-R.; Rudin, B.; Golling, M.; Unold, H. J.; Südmeyer, T.; Keller, U.

Publication date:

2007

Permanent link:

https://doi.org/10.3929/ethz-b-000005917

Rights / license:

In Copyright - Non-Commercial Use Permitted

Originally published in:

Applied Physics B 88(4), https://doi.org/10.1007/s00340-007-2760-1 
D.J.H.C. MAAS

A.-R. BELLANCOURT

B. RUDIN

M. GOLLING

H.J. UNOLD

T. SÜDMEYER

U. KELLER

\section{Vertical integration of ultrafast semiconductor lasers}

Department of Physics, Institute of Quantum Electronics, ETH Zurich, Wolfgang-Pauli-Str. 16, 8093 Zurich, Switzerland

\section{Received: 6 August 2007 \\ Published online: 22 August 2007 • (C) Springer-Verlag 2007}

ABSTRACT Lasers generating short pulses - referred to as ultrafast lasers - enable many applications in science and technology. Numerous laboratory experiments have confirmed that ultrafast lasers can significantly increase telecommunication data rates [1], improve computer interconnects, and optically clock microprocessors [2, 3]. New applications in metrology [4], supercontinuum generation [5], and life sciences with two-photon microscopy [6] only work with ultrashort pulses but have relied on bulky and complex ultrafast solid-state lasers. Semiconductor lasers are ideally suited for mass production and widespread applications, because they are based on a waferscale technology with a high level of integration. Not surprisingly, the first lasers entering virtually every household were semiconductor lasers in compact disk players. Here we introduce a new concept and make the first feasibility demonstration of a new class of ultrafast semiconductor lasers which are power scalable, support both optical and electrical pumping and allow for wafer-scale fabrication. The laser beam propagates vertically (perpendicularly) through the epitaxial layer structure which has both gain and absorber layers integrated. In contrast to edge-emitters, these lasers have semiconductor layers that can be optimized separately by using different growth parameters and with no regrowth. This is especially important to integrate the gain and absorber layers, which require different quantum confinement. A saturable absorber is required for pulse generation and we optimized its parameters with a single selfassembled InAs quantum dot layer at low growth temperatures. We refer to this class of devices as modelocked integrated external-cavity surface emitting lasers (MIXSEL). Vertical integration supports a diffraction-limited circular output beam, transformlimited pulses, lower timing jitter, and synchronization to an external electronic clock. The pulse repetition rate scales from $1-\mathrm{GHz}$ to $100-\mathrm{GHz}$ by simply changing the laser cavity length. This result holds promise for semiconductor-based high-volume wafer-scale fabrication of compact, ultrafast lasers.

PACS 42.55.Px; 42.55.Xi; 42.65.Re; 78.47.+p; 78.67.-n; 78.67.Hc

1

\section{Introduction}

The vertical external cavity surface emitting laser (VECSEL) [7] has recently gained much interest because of its power scaling potential. In a VECSEL, the light is emitted in a perpendicular direction through the epitaxial surface, in contrast to edge-emitting lasers, where the beam propagates in the epitaxial layers. An important advantage of optically pumped VECSELs is that they can convert fairly lowcost, low-beam-quality optical pump power from high-power diode laser bars into a near-diffraction-limited output beam with good efficiency, in wavelength regions that are not covered by established solid-state laser gain materials. CW output powers of up to $30 \mathrm{~W}$ with a beam quality factor $M^{2}$ of 3 have been reported from such optically pumped VECSELs [8], and electrically pumped devices have reached $0.5 \mathrm{~W}$ single transverse mode output power (i.e. $M^{2}=1.1$ ) [9]. To date, ultrafast VECSELs [10] have been successfully reported (Fig. 1) with average output powers of up to $2.1 \mathrm{~W}$ [11], pulse repetition rates of up to $50 \mathrm{GHz}$ [12] and pulse durations as short as $477 \mathrm{fs}$ [13]. All these results had diffraction-limited, circular output beams. These ultrafast VECSELs (see Fig. 2a) have at least three cavity elements, the gain structure, the output coupler and a semiconductor saturable absorber mirror (SESAM) [14, 15] for the generation of ultrashort pulses. Since VECSELs are semiconductor lasers, the integration of a semiconductor saturable absorber in the gain structure should be feasible even though a straightforward integration from the separate device elements does not work and a new concept had to be developed. We refer to this new class of devices as modelocked integrated external-cavity surface emitting lasers (MIXSELs).

Semiconductor lasers have a number of compelling advantages compared to other choices of laser material: the lasing wavelength can be tuned over a broad range by choosing the material composition, and the parameters determining the laser dynamics can be precisely engineered (e.g. recovery time, saturation energy). They are well-established in the optical communication market as reliable optical sources, and can have very low fabrication costs. So far, only ultrafast edge-emitting semiconductor lasers 


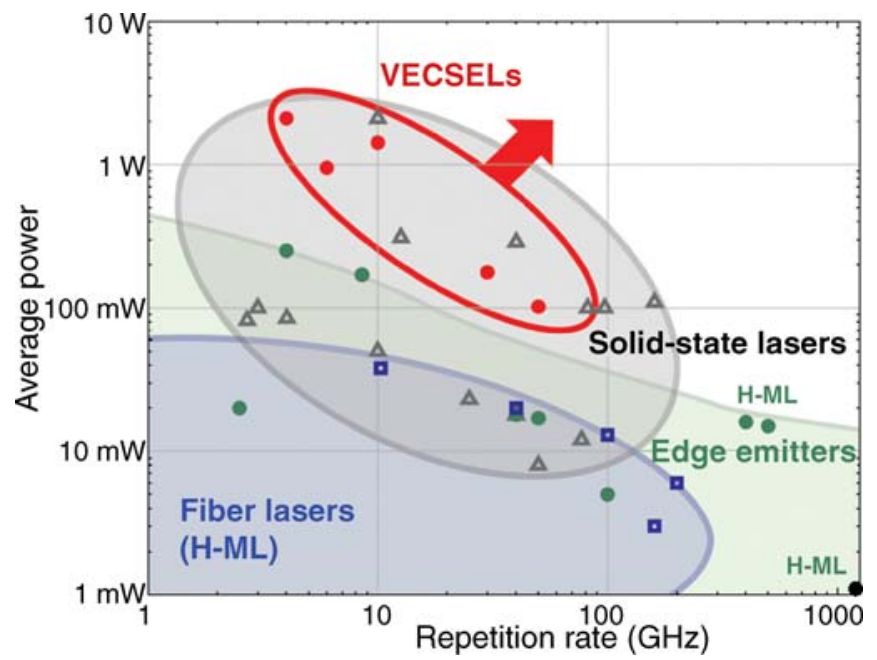

FIGURE 1 Output power for different types of ultrafast lasers operating in the gigahertz regime $\left(1 \mathrm{GHz}=10^{9} \mathrm{~Hz}\right)$. To date the available laser sources in this regime are either based on edge-emitting semiconductor lasers, harmonically modelocked (H-ML) fiber lasers, diode-pumped ion-doped solidstate lasers or vertical external cavity surface emitting semiconductor lasers (VECSELs). Above $100 \mathrm{GHz}$, the high-average-power edge-emitting lasers are also harmonically modelocked

have been fully integrated in one wafer. The highest output power to date is $250 \mathrm{~mW}$ at 4.3 GHz [16]. Unfortunately, such high average power cannot be extended to pulse repetition rates well above $10 \mathrm{GHz}$ because gain guiding at higher current densities gives rise to higher-order transverse modes. In addition, edge-emitting semiconductor lasers have strongly asymmetric beam profiles, which often need to be corrected with precisely mounted lenses. Typically, the same epitaxial layer forms both the gain (with a forward-biased section) and the saturable absorber (with a reverse-biased section) - and can therefore not be optimized independently. The long interaction length in the device introduces significant dispersion and nonlinearities. It is also challenging to fabricate an edge-emitting laser cavity length with a very precise pulse repetition rate and have this laser synchronized to an external reference clock.

Other interesting ultrafast lasers are either based on diode-pumped solidstate lasers or fiber lasers. Solid-state lasers modelocked with SESAMs have increased pulse energy by three orders of magnitude, to more than $10 \mu \mathrm{J}$ at multi-MHz repetition rates, increased pulse repetition rates by more than two orders of magnitude to $160 \mathrm{GHz}$, and reduced pulse duration into the sub-10femtosecond regime [17-19]. However, gain material and absorber material are different and can not be manufactured simultaneously, which results in higher complexity and costs. Moreover, direct electrical pumping is not feasible. Modelocked fiber lasers are not fundamentally modelocked at high repetition rates. Instead, harmonic modelocking (H-ML) is used, where multiple pulses simultaneously circulate in the cavity at once, which is susceptible to pulse drop out and higher noise levels.

The MIXSEL is a fundamentally modelocked laser, in which only one pulse is circulating in the resonator (Fig. 2b). Every time the pulse hits a partially transmissive mirror, the output coupler, a small fraction is transmitted. The output beam consists of an equidistant pulse train with a period defined by the round-trip time of a pulse inside the laser cavity. The gain section inside the MIXSEL semiconductor structure amplifies the pulse, and a saturable absorber section initiates and stabilizes the pulse formation. Such a saturable absorber has a constant loss for cw-lasing, whereas pulses with high peak powers are able to saturate the absorber and therefore reduce its loss.

The semiconductor gain structure (Fig. 2a) contains a highly reflective (HR) mirror, a gain section, and an antireflective (AR) coating which provides low transmission loss from the semiconductor device to the external cavity. Its gain section contains quantum wells (QWs), thin layers (for example, $10 \mathrm{~nm}$ ) of semiconductors surrounded by barrier materials with larger potential en-
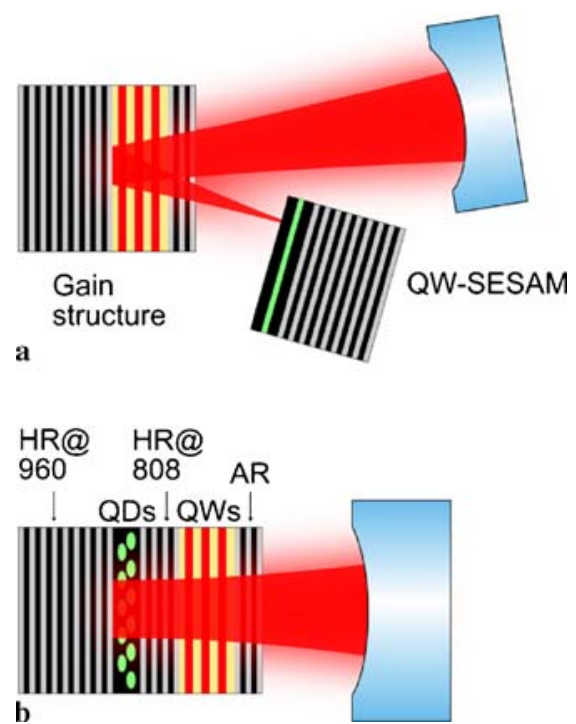

FIGURE 2 Integration scheme, progressing from conventional VECSEL-SESAM modelocking with (a) large mode area ratios and thus large cavities, (b) to absorber-gain integration in a modelocked integrated external-cavity surface emitting laser (MIXSEL). The MIXSEL semiconductor wafer structure contains two high reflectors (HR), quantum dot (QD) saturable absorber, quantum well (QW) gain and an anti-reflective (AR) coating. The first HR reflects the laser light and forms the laser cavity together with the external output coupler. The second one, the intermediate $\mathrm{HR}$, is to prevent the pump light bleaching the saturable absorber

ergy (i.e. a larger bandgap) that confine electrons and holes inside the QWs. The gain structure is used as an amplifying mirror mounted onto a heat sink. Because the beam diameters are typically larger than the total device thickness, the gain structure has an almost perfect one-dimensional heat flow, which allows power scaling: doubling the mode size doubles the output power [20] but maintains approximately the same temperature rise in the pumped material. The mode size can be easily adjusted with the output coupler.

The semiconductor saturable absorber is responsible for self-starting and maintaining modelocking [17]. To date, the saturable absorber has been integrated into a separate mirror structure device (i.e. the SESAM) and typically used QW-absorbers with a bandgap slightly smaller than the photon energy to absorb the intracavity light and generate a self-amplitude modulation (saturable absorption). When most possible states for the excited electrons are occupied, the absorption becomes saturated which then reduces the loss. A SESAM has three important param- 


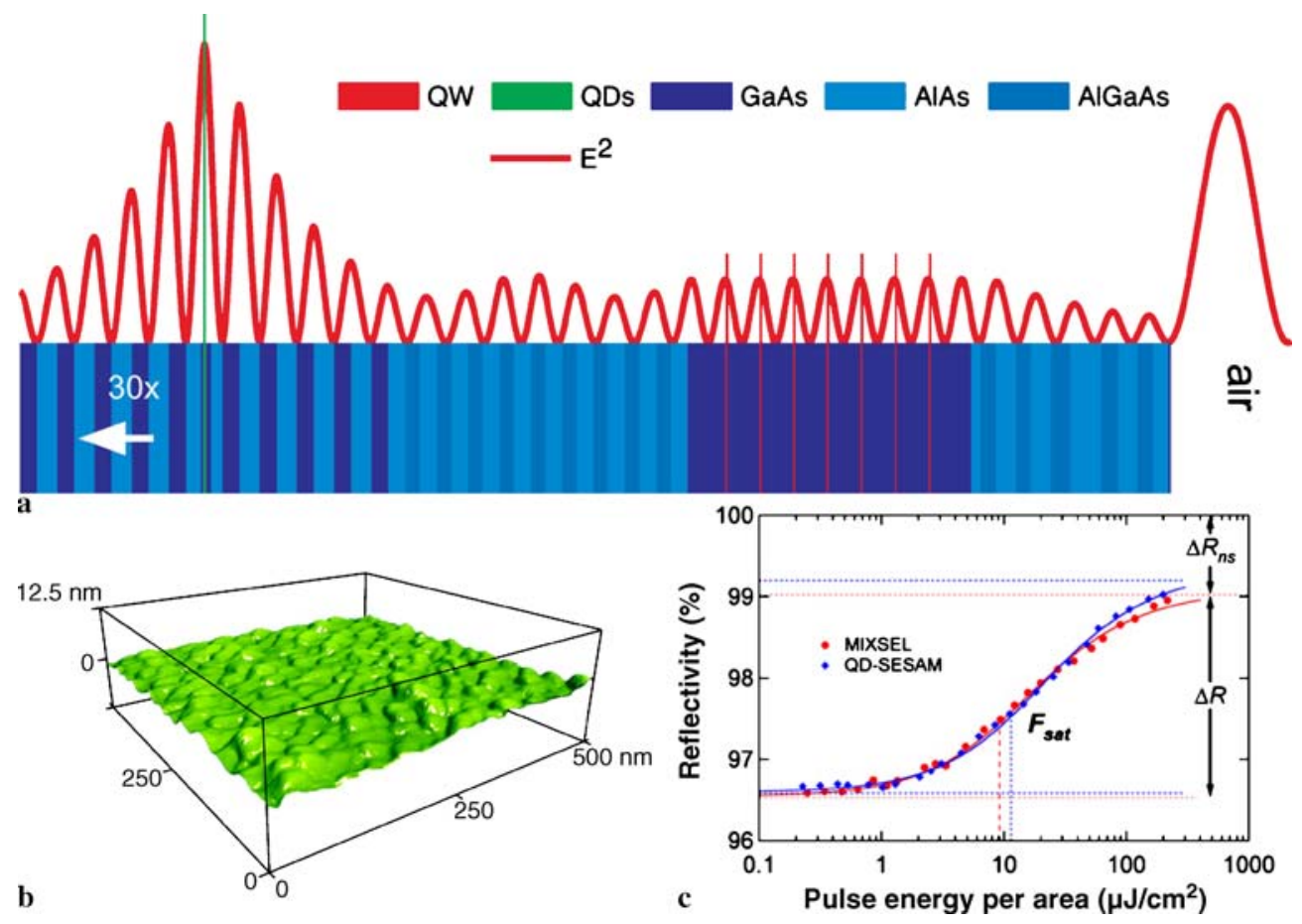

FIGURE 3 MIXSEL design. (a) Simulated electric field intensity inside the MIXSEL structure. The QD absorber layer is integrated in a "resonant structure" which means that we have an $E$-field enhancement in the absorber layer compared to the gain layers. The structure is grown with molecular beam epitaxy (MBE) on a GaAs substrate and is approximately $8 \mu \mathrm{m}$ thick (see Sect. 2 for more details). (b) AFM topography of the QD absorber layer (measured with a standard AFM tip radius of $10 \mathrm{~nm}$ ) grown under the same conditions as in the MIXSEL but without any cover layer and without annealing (i.e. QD layer was grown at $430{ }^{\circ} \mathrm{C}$ with $\approx 2$ monolayer InAs deposition). (c) Nonlinear reflectivity measurement at a center wavelength of 957 nm at room temperature of an annealed resonant QD-SESAM (blue) and a full MIXSEL (red) with the same QD layer (in both cases grown at $400{ }^{\circ} \mathrm{C}$ ). Under these conditions, the quantum wells in the MIXSEL structure did not absorb. We measured a saturation fluence $F_{\text {sat }}$ of $9 \mu \mathrm{J} / \mathrm{cm}^{2} \mathrm{resp} .11 \mu \mathrm{J} / \mathrm{cm}^{2}$, a modulation depth $\Delta R$ of $2.5 \%$ resp. $2.6 \%$ and a nonsaturable loss $\Delta R_{\mathrm{ns}}$ of $1 \%$ resp. $0.9 \%$ for the MIXSEL resp. QD-SESAM. The measurement wavelength was slightly detuned from resonance with a lower electric field enhancement in the absorber (i.e. $\approx 2.5$ instead of 4 ). Here we clearly show that the additional growth of the MIXSEL structure does not degrade the saturable absorber parameters which would result in a significant increase in nonsaturable loss $\Delta R_{\mathrm{ns}}$

eters: the modulation depth, which is the difference in reflectivity between an unsaturated and a completely saturated SESAM, the saturation energy, which is the pulse energy needed to obtain a roll-over of the reduction in loss with higher pulse energies, and the nonsaturable absorption, which introduces a residual loss even when the absorber is fully saturated. The saturation energy depends on the mode size; if the area on the SESAM that we have to saturate becomes smaller, we need less pulse energy to saturate it. Highpower ultrafast VECSELs are typically based on QW-SESAMs, with properties similar to the QWs in the gain structure, which means similar saturation conditions. For stable modelocking, the absorber needs to saturate earlier and recover faster than the gain [19], and we have to choose a smaller spot size on the SESAM than on the gain structure (Fig. 2a). However, integration of such a QW absorber into the gain structure (Fig. 2b) is not possible due to the different mode areas.
With quantum dot (QD) saturable absorbers, we can resolve the saturation issue. Compared to QWs, QDs confine the electrons in all three dimensions (and are therefore referred to as dots). This reduces the density of states and can lower the required optical intensity for band filling if the oscillator strength does not change. In addition, with the dot density and the optical design of the structure (moving from an antiresonant to a resonant design, see Fig. 3a) [21] we can further reduce the saturation energy and keep the modulation depth to the $1 \%$-level. This is not possible with quantum well absorbers, because the required reduction of the saturation fluence by a resonant design would cause a too large increase of modulation depth (up to 11 times, see [21]).

Until recently QDs have been mainly optimized for different applications, such as for example for single-photon emitters [22], for low-threshold emitters [23], for multi-QD-layers SESAMs [24], for cavity quantum electrodynamics [25] or for quantum com- putations [26]. Here we operate in a completely different regime because we used low temperature (LT) $\mathrm{MBE}$ growth to obtain a fast recovery time. LT growth incorporates defect states that act as efficient traps for the excited electrons and therefore reduce their recombination time (i.e. the recovery time of the saturable absorber). Normally self-assembled InAs QDs on GaAs are grown at a growth temperature of 500 to $550{ }^{\circ} \mathrm{C}$ and due to the $7 \%$ lattice mismatch between GaAs and InAs, the two-dimensional growth changes into a three-dimensional one after a deposition of about 1.5 to 1.7 monolayers of InAs [27]. We typically grow our QD saturable absorbers at around $400{ }^{\circ} \mathrm{C}$ and a thickness of about two monolayers of InAs is sufficient for the formation of quantum dots (Fig. 3b). In addition, at lower growth temperatures the mobility of the In and As atoms on the GaAs surface is strongly reduced which lowers the material thickness threshold for QD formation. Such a QD saturable absorber layer is integrated into 

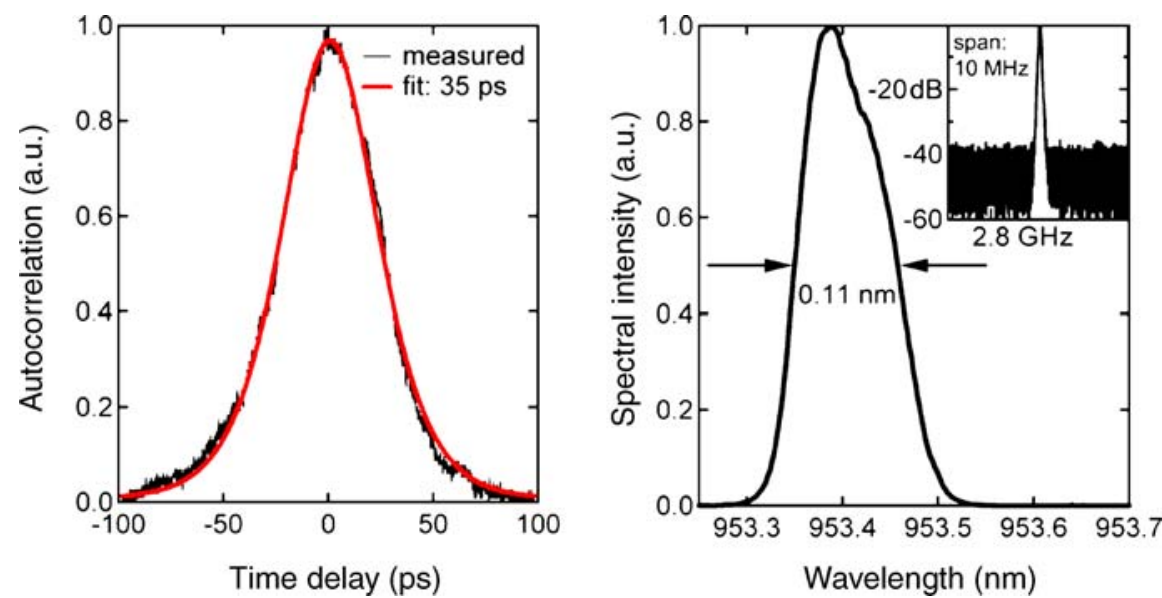

FIGURE 4 Pulse characterization of the current result with $40 \mathrm{~mW}$ average output power. Left: autocorrelation with $\operatorname{sech}^{2}$-fit, right: optical spectrum, inset: microwave spectrum on a $10-\mathrm{MHz}$ span with $100-\mathrm{kHz}$ resolution bandwidth

the MIXSEL structure (Fig. 3a) with a stronger electric field enhancement in the QD absorber layer compared to the QW gain layers - we refer to this as the "resonant" design motivated by our previous SESAM design work [21]. This reduces the saturation fluence $F_{\text {sat }}$ of the saturable absorber to well below $10 \mu \mathrm{J} / \mathrm{cm}^{2}$ (Fig. 3c is measured slightly off resonance - at resonance we would obtain an even smaller saturation fluence). In comparison a standard QW-SESAM in a nonresonant structure typically has a saturation fluence well above $10 \mu \mathrm{J} / \mathrm{cm}^{2}$ into the $100 \mu \mathrm{J} / \mathrm{cm}^{2}$ regime [21]. Both QW- and QD-SESAMs have a recovery time in the picosecond regime.

The development of the QD saturable absorber was the crucial step towards the MIXSEL. The MIXSEL cavity is shown in Fig. 2b, which is not to scale. The semiconductor device only adds about $10 \mu \mathrm{m}$ to the laser cavity length - so most of the laser cavity beam propagates in air (e.g. a $50 \mathrm{GHz}$ pulse repetition rate corresponds to a cavity length of $3 \mathrm{~mm}$ ). This could be replaced with another transparent wafer with the semiconductor wafer glued to one side and an integrated curved output coupler on the other side [28]. We used an output coupler with a radius of curvature of $60 \mathrm{~mm}$. The heat sink is cooled down to $-10^{\circ} \mathrm{C}$ using two peltier elements. The thick (i.e. $600 \mu \mathrm{m}$ ) substrate has a poor thermal conductivity, resulting in a high temperature increase (over $110^{\circ} \mathrm{C}$ ), ultimately limiting the output power. This problem also arises with VECSELs and is usu- ally solved by growing the layers in reverse order and substrate removal (i.e. "upside-down" structure) [8, 20]. Without the thick substrate, the temperature increase can be reduced (below $20^{\circ} \mathrm{C}$ ) allowing for substantially higher output power.

The cw pump source delivered $1.5 \mathrm{~W}$ at $808 \mathrm{~nm}$ and is focused to a circular pump spot with a radius of $80 \mu \mathrm{m}$. The lasing wavelength can be tuned between $953 \mathrm{~nm}$ and $956 \mathrm{~nm}$ with an intracavity $25 \mu \mathrm{m}$-thick fused silica etalon. This gives us some adjustments in the field enhancement ratio between absorber and gain and the cavity group delay dispersion. Stable modelocking was obtained with a 5.4-cm long straight cavity and a $0.35 \%$ transmission output coupler. The laser delivered $40 \mathrm{~mW}$ average power in $35 \mathrm{ps}$ pulses at $2.8 \mathrm{GHz}$ with a spectral full-width half-maximum (FWHM) of $0.11 \mathrm{~nm}$ (Fig. 4). Modelocking was also observed without the etalon but with less stability. The limited output power and low optical-to-optical efficiency is caused by the high temperature increase. Power, pulse duration, and pulse repetition rates can be improved in the future with an "upside-down" structure based on the previous results with modelocked VECSELs.

With the "upside-down" structure, we have even less stringent growth requirements for the MIXSEL. In the current "right-side-up" growth, the QW gain section has to be grown after the QD layer grown at low temperature, which introduces some defects. "Upside-down" growth would reduce such defects and enhance gain.
However, with the current "rightside-up" growth no further processing for laser testing was required and we could emphasize our efforts on the understanding and optimization of the QD saturable absorber layer which was the crucial step towards the feasibility demonstration of our integration concept. Currently we still have some growth inaccuracies which cause a large group delay dispersion that is responsible for the longer chirped pulses. In-situ growth characterization can be used to obtain higher accuracy.

In conclusion, we have successfully demonstrated the first passively modelocked VECSEL with both the gain and absorber integrated into a single semiconductor structure. This new-class of passively modelocked semiconductor lasers could enable the realization of robust, ultra-compact multi-GHz sources suitable for mass production. The simple straight cavity geometry allows for very high repetition rates. Moreover, advanced heat management will result in multi-Watt average output powers as previously demonstrated in the former VECSEL-SESAM results [10]. The last step towards even lower-cost and more compact ultrafast semiconductor surface emitting lasers will be electrical pumping. This would result in devices ideally suited for many applications such as telecommunications, optical clocking, frequency metrology, microscopy, laser display - anywhere where the current ultrafast laser technology is considered to be too bulky or expensive.

\section{$2 \quad$ Methods \\ 2.1 MIXSEL design and growth}

The QDs are grown at $430^{\circ} \mathrm{C}$, the QWs at $520^{\circ} \mathrm{C}$, and the rest of the structure is grown at $600{ }^{\circ} \mathrm{C}$ for less defects and high optical gain. This higher temperature does not affect the QWs, however it anneals the QDs. This annealing changes the dot composition and size, resulting in a blue shift of the absorption and emission wavelength [29]. We could compensate for this by initially growing the QDs at a photoluminescence peak of $1100 \mathrm{~nm}$, then annealing the devices to shift the absorption peak to around $950 \mathrm{~nm}$ to match our lasing wavelength. The rest of the structure is standard VECSEL 
growth: the intermediate distributed Bragg reflector (DBR) consists of AlAs and $\mathrm{Al}_{0.2} \mathrm{Ga}_{0.8} \mathrm{As}$, where both materials do not absorb the pump light. The bottom DBR consists of 30 pairs of AlAs/GaAs. The gain section consists of seven $\mathrm{In}_{0.13} \mathrm{Ga}_{0.87}$ As quantum wells with a thickness of $7 \mathrm{~nm}$ surrounded by GaAs barriers that absorb the pump light. Finally, the anti-reflective coating has the same material compositions as the intermediate DBR. In this resonant structure we embedded the QD-layer inside a $\lambda / 2$-thick "sub-cavity" inside the bottom DBR which gives the field enhancement in the absorber layer. The QDs are embedded inside a 20-nm thick GaAs layer surrounded by AlAs with a total optical thickness of $\lambda / 2$ (Fig. 3a). We kept the GaAs-layer as thin as possible to minimize residual absorption in GaAs. After the growth, we solder the sample to a copper heatsink.

\section{$2.2 \quad Q D-S E S A M$ design}

In Fig. 3c we show for comparison the nonlinear reflectivity measurement of a resonant QD-SESAM for which we used the same growth conditions as for the MIXSEL structure, except that we used a slightly lower growth temperature of $400^{\circ} \mathrm{C}$. This QDSESAM was again a resonant design with the $\lambda / 2$ "sub-cavity" on top and the QD-layer embedded inside the thin $\mathrm{GaAs}$ (i.e. $\approx 20 \mathrm{~nm}$ ) and thicker AlAs layers. After the growth, the structure was in-situ annealed for $5 \mathrm{~h}$ at $600^{\circ} \mathrm{C}$, which is similar to the annealing that occurs during the MIXSEL structure growth. Within the first hour of the annealing process, the PL wavelength changes by a total of $125 \mathrm{~nm}$, then it changes linearly with time at $5 \mathrm{~nm} / \mathrm{h}$.

\subsection{Nonlinear reflectivity measurements}

The measured nonlinear reflectivity of the MIXSEL and the QDSESAM is shown in Fig. 3c. The details of the measurement set-up are described by Haiml et al. [30]. For this measurement we used a passively modelocked VECSEL with a separate SESAM in a V-shaped cavity (Fig. 2a) with a pulse duration of $6 \mathrm{ps}$, a maximum average output power of $2 \mathrm{~W}$ and a pulse repetition rate of $1.5 \mathrm{GHz}$. The laser was chopped and focused to a spot radius of $5 \mu \mathrm{m}$ on the device under test and the center wavelength was at $957 \mathrm{~nm}$.

ACKNOWLEDGEMENTS This work was supported in part by the Intel Corporation through a university sponsored research agreement, the European Network of Excellence "ePIXnet," and ETH Zurich with the FIRST clean-room facility.

AUTHOR INFORMATION The first three authors contributed equally to this work.

\section{REFERENCES}

1 L.F. Mollenauer, P.V. Mamyshev, J. Gripp, M.J. Neubelt, N. Mamysheva, L. GrunerNielsen, T. Veng, Opt. Lett. 25, 704 (2000)

2 M.J. Kobrinsky, B.A. Block, J.-F. Zheng, B.C. Barnett, E. Mohammed, M. Reshotko, F. Robertson, S. List, I. Young, K. Cadien, Intel Technol. J. 8(2), 129 (2004), available at http://www.intel.com/technology/itj/ archive.htm

3 A. Bhatnagar, C. Debaes, R. Chen, N.C. Helman, G.A. Keeler, D. Agarwal, H. Thienpoint, D.A.B. Miller, "Receiverless clocking of a CMOS digital circuit using short optical pulses," presented at 2002 IEEE/LEOS Annual Meeting; Conference Proceedings, 15th Annual Meeting of the IEEE Lasers \& Electro-Optics Society, Glasgow, Scotland, 2002

4 Th. Udem, R. Holzwarth, T.W. Hänsch, Nature 416, 233 (2002)

5 P. Russell, Science 299, 358 (2003)

6 K. Ohki, S. Chung, Y.H. Ch'ng, P. Kara, R.C. Reid, Nature 433, 597 (2005)

7 M. Kuznetsov, F. Hakimi, R. Sprague, A. Mooradian, IEEE Photon. Technol. Lett. 9, 1063 (1997)

8 J. Chilla, S. Butterworth, A. Zeitschel, J. Charles, A. Caprara, M. Reed, L. Spinelli, "High Power Optically Pumped Semiconductor Lasers," presented at Photonics West 2004, Solid State Lasers XIII: Technology and Devices, in Proc. SPIE 5332, 2004

9 J.G. McInerney, A. Mooradian, A. Lewis, A.V. Shchegrov, E.M. Strzelecka, D. Lee, J.P. Watson, M. Liebman, G.P. Carey, B.D. Cantos, W.R. Hitchens, D. Heald, Electron. Lett. 39, 523 (2003)
10 U. Keller, A.C. Tropper, Phys. Rep. 429, 67 (2006)

11 A. Aschwanden, D. Lorenser, H.J. Unold, R. Paschotta, E. Gini, U. Keller, Opt. Lett. 30, 272 (2005)

12 D. Lorenser, D.J.H.C. Maas, H.J. Unold, A.R. Bellancourt, B. Rudin, E. Gini, D. Ebeling, U. Keller, IEEE J. Quantum Electron. QE-42, 838 (2006)

13 A. Garnache, S. Hoogland, A.C. Tropper, I. Sagnes, G. Saint-Girons, J.S. Roberts, Appl. Phys. Lett. 80, 3892 (2002)

14 U. Keller, D.A.B. Miller, G.D. Boyd, T.H. Chiu, J.F. Ferguson, M.T. Asom, Opt. Lett. 17, 505 (1992)

15 U. Keller, K.J. Weingarten, F.X. Kärtner, D. Kopf, B. Braun, I.D. Jung, R. Fluck, C. Hönniger, N. Matuschek, J. Aus der Au, IEEE J. Sel. Top. Quantum Electron. 2, 435 (1996)

16 J.J. Plant, J.T. Gopinath, B. Chann, D.J. Ripin, R.K. Huang, P.W. Juodawlkis, Opt. Lett. 31, 223 (2006)

17 U. Keller, Nature 424, 831 (2003)

18 G. Steinmeyer, D.H. Sutter, L. Gallmann, N. Matuschek, U. Keller, Science 286, 1507 (1999)

19 U. Keller, Prog. Opt. 46, 1 (2004)

20 R. Häring, R. Paschotta, A. Aschwanden, E. Gini, F. Morier-Genoud, U. Keller, IEEE J. Quantum Electron. QE-38, 1268 (2002)

21 G.J. Spühler, K.J. Weingarten, R. Grange, L. Krainer, M. Haiml, V. Liverini, M. Golling, S. Schön, U. Keller, Appl. Phys. B 81, 27 (2005)

22 C. Santori, D. Fattal, J. Vuckovic, G.S. Solomon, Y. Yamamoto, Nature 419, 594 (2002)

23 H.Y. Liu, D.T. Childs, T.J. Badcock, K.M. Groom, I.R. Sellers, M. Hopkinson, R.A. Hogg, D.J. Robbins, D.J. Mowbray, M.S. Skolnick, "High-performance threelayer 1.3-mu m InAs-GaAs quantum-dot lasers with very low continuous-wave roomtemperature threshold currents," IEEE Photon. Technol. Lett. 17, 1139 (2005)

24 E.U. Rafailov, S.J. White, A.A. Lagatsky, A. Miller, W. Sibbett, D.A. Livshits, A.E. Zhukov, V.M. Ustinov, IEEE Photon. Technol. Lett. 16, 2439 (2004)

$25 \mathrm{~K}$. Hennessy, A. Badolato, M. Winger, D. Gerace, M. Atatüre, S. Gulde, S. Fält, E.L. Hu, A. Imamoglu, Nature 445, 896 (2007)

26 Xiaoqin Li, Yanwen Wu, D.G. Steel, D. Gammon, T.H. Stievater, D.S. Katzer, D. Parker, C. Piermarocchi, L.J. Sham, Science 301, 809 (2003)

27 R. Leon, P.M. Petroff, D. Leonard, S. Fafard, Science 267, 1966 (1995)

28 M.W. Wiemer, R.I. Aldaz, D.A.B. Miller, J.S. Harris, IEEE Photon. Technol. Lett. 17, 1366 (2005)

29 S. Malik, C. Roberts, R. Murray, M. Pate, Appl. Phys. Lett. 71, 1987 (1997)

30 M. Haiml, R. Grange, U. Keller, Appl. Phys. B 79, 331 (2004) 